\title{
Relations entre le pouvoir minéralisateur des sols et la minéralisation nette de l'azote au champ
}

\author{
JE Delphin, JY Chapot, A Schoellen \\ avec la collaboration technique de C Huck, C Schneider et G Schwab
}

INRA, station de recherches grandes cultures, laboratoire d'agronomie, 28, rue de Herrlisheim, 68021 Colmar Cedex, France

(Reçu le 4 juillet 1990; accepté le 28 mars 1991)

\begin{abstract}
Résumé - Le pouvoir minéralisateur de 6 sols de la plaine d'Alsace est comparé à la minéralisation au champ pendant une culture de maïs. Le pouvoir minéralisateur est estimé par des tests d'incubation conduits à humidité constante et à $20^{\circ} \mathrm{C}$ ou à la température extérieure. L'estimation de la minéralisation nette au champ est obtenue par 2 méthodes : bilan d'azote minéral en parcelle nue et bilan de l'azote exporté par un maïs sans fumure N. Le rapport entre l'azote minéral disponible au champ (reliquat + minéralisation) sur parcelle nue et l'azote mobilisé par les parties aériennes varie pour 4 parcelles de 1,2 à 1,5. Pour 2 autres parcelles, ce rapport est plus faible et laisse supposer une sous-estimation de la minéralisation par la méthode de la parcelle nue. La comparaison des résultats de laboratoire aux mesures de terrain fait apparaître, pour 3 parcelles, un minéralisation estimée au champ largement inférieure aux potentialités. Si la lixiviation des nitrates peut en partie en être la cause, l'état physique du sol au champ (humidité, porosité), par ses effets sur l'activité microbienne, est vraisemblablement également impliqué.
\end{abstract}

azote / pouvoir minéralisateur / test d'incubation / bilan d'azote / Zea mays = maïs

Summary - Relationship between N mineralization capacity and net nitrogen mineralization in field conditions. The net nitrogen mineralization capacity of 6 soils of the Alsace Plain (France) was compared to available $N$ measured in maize fields. The nitrogen mineralization potential of soils was assessed by incubation tests with constant water content (90\% of water content at $0.1 \mathrm{MPa}$ matric suction) at $20^{\circ} \mathrm{C}$ and at outdoor temperatures. Two field procedures were used to estimate the $N$ mineralization: balance-sheet of inorganic $N$ on bare soil and of $N$ uptake by maize receiving no $N$ fertilizer (table II). The ratio between inorganic $N$ available on bare soil and $N$ uptake by aerial parts of the culture ranged between 1.2-1.5 in 4 plots but was lower in 2 plots; one possible explanation was an underestimation of $N$ availability by bare soil procedure. Mineralization $N$ evaluation by field procedures in 3 plots was largely lower than the estimate by biological test incubation (fig 3). These results were likely due to $N$ leaching and to the effect of soil water content and air porosity on microbial activity in field conditions.

nitrogen / mineralization capacity / incubation test / nitrogen balance-sheet / maize

\section{INTRODUCTION}

L'affinement du calcul de la fertilisation azotée des cultures est un moyen de réduire le gaspillage d'azote minéral en agriculture et de limiter le préjudice que ces excès peuvent causer à la qualité des eaux souterraines et superficielles. Le raisonnement de la fumure azotée des cultures de printemps achoppe principalement en raison d'une méconnaissance de la quantité d'azote minéral fournie par le sol, à partir de matières organiques variées (Taureau et Desvignes, 1990). Cette fourniture intervient directement pendant le cycle cultural, elle contribue également au reliquat d'azote minéral avant culture.

Pour les matières organiques humifiées, des travaux de laboratoire récents ont permis de mieux cerner les principaux facteurs édaphiques qui contrôlent la minéralisation et de fournir une estimation satisfaisante du pouvoir minéralisateur des sols agricoles à partir de leurs caractéristiques physico-chimiques (Delphin, 1986; Chaussod et al, 1986). On connaît mal en revanche l'expression au champ du pouvoir minéralisateur estimé en laboratoire. De nombreuses études ont porté sur l'estimation de la minéralisation au 
champ, mais ces résultats sont rarement confrontés aux potentiels fournis par les tests d'incubation (Meisinger, 1984). Mary et Rémy (1979) observent une corrélation significative entre le potentiel de minéralisation et la minéralisation du sol sous une culture de blé d'hiver. Dans une autre expérimentation, ces mêmes liaisons apparaissent moins nettes (Mary, 1988). L'estimation de la minéralisation au champ se heurte, en effet, à des difficultés méthodologiques liées à la diversité des processus impliqués dans l'évolution de l'azote et à l'hétérogénéité de sa répartition dans le sol.

Les résultats présentés ici portent sur la comparaison entre le pouvoir minéralisateur de quelques sols agricoles estimé en conditions contrôlées et la minéralisation déterminée directement au champ sur la période de développement du maïs, afin d'établir une correspondance entre ces 2 mesures et de mettre en évidence d'éventuels obstacles à la réalisation in situ des potentialités.

\section{MATÉRIEL ET MÉTHODES}

L'étude porte sur 6 sols agricoles de la plaine d'Alsace (France) dont quelques caractéristiques figurent au tableau I.

\section{Tests d'incubation}

Les échantillons de sols ont été prélevés début avril dans la couche arable $(0-20 \mathrm{~cm})$ et conservés en état à $5^{\circ} \mathrm{C}$ pendant $10-15 \mathrm{j}$. Les prélèvements sont effectués 5 mois au moins après l'enfouissement des résidus de la culture précédente; on peut supposer que, passé ce laps de temps, la minéralisation des matières organiques fraîches pèse peu sur les fournitures d'azote par le sol. Des essais au champ montrent, en effet, que les fractions facilement dégradables (environ $80 \%$ des résidus de récolte), ont des demi-vies courtes de l'ordre de 2 mois (Jenkinson et Rayner, 1977). Quoiqu'il en soit, la minéralisation résiduelle est prise en compte ultérieurement dans les bilans d'azote minéral des essais en pots et au champ.

Les incubations conduites en laboratoire à $20^{\circ} \mathrm{C}$ $\pm 0,5$ portent sur $1,2 \mathrm{~kg}$ de terre fraîche (environ $1 \mathrm{~kg}$ de terre sèche), préalablement homogénéisée, dans des pots cylindriques de $0,01 \mathrm{~m}^{2}$ de section et de 13 $\mathrm{cm}$ de hauteur (4 répétitions). L'humidité est maintenue à $90 \%$ de l'humidité équivalente $(\mathrm{pF}=3)$ en corrigeant régulièrement les pertes d'eau. Des pots sont sacrifiés après 25,150 et 300 j d'incubation pour la mesure de l'azote minéral. L'azote minéral produit entre le $25^{\mathrm{e}}$ et le $300^{\mathrm{e}}$ j d'incubation, définissant le pouvoir minéralisateur des sols (Delphin, 1986), est pris ici comme indicateur de potentialités.

Une expérience est également menée à l'extérieur dans des pots de même section et de $30 \mathrm{~cm}$ de hauteur, enterrés et couverts pour les protéger des pluies. Ces pots mis en place début mai contiennent $3,3 \mathrm{~kg}$ de terre fraîche maintenue à $90 \%$ de l'humidité équivalente par des apports hebdomadaires d'eau compensant l'évaporation. Cinq pots prélevés entre la date de semis du maïs et l'hiver servent au suivi de l'azote minéral. Les températures mensuelles moyennes du sol à $10 \mathrm{~cm}$ durant l'essai ont varié de $16,1^{\circ} \mathrm{C}$ en mai à $21,1^{\circ} \mathrm{C}$ en juillet et $2,8^{\circ} \mathrm{C}$ en décembre, la moyenne sur la durée de l'expérience étant de $14,3^{\circ} \mathrm{C}$. La densité sèche des échantillons de sols conduits en incubation est faible $\left(1,1-1,2 \mathrm{~g} \cdot \mathrm{cm}^{-3}\right)$.

\section{Méthodes au champ}

Deux méthodes d'estimation de la minéralisation endogène nette au champ ont été adoptées : suivi de l'azote minéral sous sol nu et exportation d'azote par

Tableau I. Quelques caractéristiques physico-chimiques de la couche arable $(0-20 \mathrm{~cm})$ des 6 parcelles.

\begin{tabular}{|c|c|c|c|c|c|c|c|c|}
\hline & \multirow[b]{2}{*}{ Argiles } & \multirow[b]{2}{*}{ Limons } & \multicolumn{2}{|c|}{ Teneurs ( $g / k g)$} & \multirow[b]{2}{*}{$N$ total } & \multirow[b]{2}{*}{ Calcaire } & \multirow[t]{2}{*}{$p H$} & \multirow[t]{2}{*}{ Profondeur $(m)$} \\
\hline & & & Sables & C organique & & & & \\
\hline \multicolumn{9}{|l|}{ Limons calcaires } \\
\hline Site $\mathrm{n}^{\circ} 1$ (Colmar) & 225 & 659 & 95 & 12,1 & 1,33 & 160 & 8,3 & $>1,2$ \\
\hline Site $n^{\circ} 2$ (Epfig) & 221 & 667 & 91 & 12,4 & 1,40 & 1 & 7,5 & $>1,2$ \\
\hline \multicolumn{9}{|l|}{ Alluvions du Rhin } \\
\hline Site $n^{\circ} 3$ (Dessenheim) & 257 & 381 & 343 & 11,4 & 1,32 & 187 & 8,3 & 1,0 \\
\hline Site $n^{\circ} 4$ (Weckolsheim) & 240 & 405 & 355 & 11,2 & 1,28 & 274 & 8,3 & 1,0 \\
\hline \multicolumn{9}{|l|}{ Alluvions de l'III } \\
\hline Site $n^{\circ} 5$ (Oberhergheim) & 230 & 394 & 360 & 9,0 & 1,02 & 1 & 6,7 & $>1,2$ \\
\hline Site $n^{\circ} 6$ (Niederhergheim) & 287 & 278 & 412 & 12,9 & 1,39 & 1 & 6,4 & 0,8 \\
\hline
\end{tabular}


une culture de maiis sans fumure azotée. Le choix des parcelles vise principalement des sols profonds et l'absence d'amendements organiques récents autres que les résidus de la culture qui précède afin de réduire les sources d'erreur (lixiviation, minéralisation exogène).

Les parcelles nues $(20 \times 13 \mathrm{~m})$ sont subdivisées en 4 blocs $(3 \times 2 \mathrm{~m})$ au sein desquels, à chaque date de prélèvement, sont réalisés 4 forages de la tarière sur $1,2 \mathrm{~m}$ de profondeur de 0,2 en $0,2 \mathrm{~m}$, chaque horizon servant à constituer un prélèvement moyen. Seize carottages par date permettent d'estimer avec une précision satisfaisante le stock moyen d'azote minéral d'une parcelle (Lindemann, 1986). Pour améliorer cette précision, les prélèvements de début et de fin du bilan (tableau II) ont été répétés à 10-15 j d'intervalle. Afin de limiter les transferts de nitrates en profondeur, une bâche plastique a été posée sur le sol lors de quelques irrigations. Les parcelles 1 et 2 n'ont pas été irriguées. Compte tenu des réductions d'irrigation obtenues par le bâchage, les parcelles 3 à 6 ont reçu respectivement $50,125,100$ et $100 \mathrm{~mm}$ entre fin juin et août. Les précipitations au cours de l'essai sont de $240 \mathrm{~mm}$. Les teneurs en eau des horizons de surface $(0-40 \mathrm{~cm})$ varient, selon les conditions, de la saturation à $80 \%$ de l'humidité équivalente.

Sur les parcelles maïs $0 \mathrm{~N}(40 \times 13 \mathrm{~m})$, la variété semée est Dea (8-10 pieds $/ \mathrm{m}^{2}$ ) après une culture de maïs (parcelles 1, 2, 5 et 6 ) ou de blé d'hiver (parcelles 3 et 4) dont les résidus de récolte ont été enfouis. Cent $\mathrm{mm}$ d'eau d'irrigation ont été apportés au maïs de la parcelle 3 et $150 \mathrm{~mm}$ dans les parcelles 4,5 et 6 . La production de matière sèche moyenne (tiges, feuilles et épis) est estimée au "stade ensilage» par 4 prélèvements linéaires de $10 \mathrm{~m}, 4$ échantillons représentatifs de 3 pieds servant à la détermination des teneurs en azote et de l'humidité. Le reliquat d'azote minéral dans le sol, sous maïs, après récolte, est établi de la même façon que pour les parcelles nues. Les mesures de la densité sèche des différents horizons des profils permettent d'exprimer les résultats à l'hectare.

\section{Mesures et analyses}

Les densités sèches sur les profils sont mesurées au moyen d'une sonde gamma et confirmées pour les 60 premiers $\mathrm{cm}$ par un densitomètre à membrane. L'azote minéral est extrait au $\mathrm{KCl} \mathrm{N}\left(50 \mathrm{~g}\right.$ de terre $\left./ 100 \mathrm{~cm}^{3}\right)$, $\mathrm{NO}_{3}$ et $\mathrm{NH}_{4}+$ étant dosés par colorimétrie. L'azote total (sol et plante) est déterminé selon la méthode Kjeldahl.

En l'absence de pertes (lixiviation, dénitrification), l'azote minéralisé $N_{\min }\left(\mathrm{kg} \cdot h \mathrm{a}^{-1}\right)$ au champ peut être estimé comme suit :

sur parcelle nue

$$
N_{\min }=N_{\text {mfin }}-N_{\text {mdeb }}
$$

sous maïs

$$
\mathrm{N}_{\min }=(R+1) \mathrm{N}_{\text {exp }}+\mathrm{N}_{\text {mmais }}-\mathrm{N}_{\text {mdeb }}
$$

$N_{\text {mfin }}, N_{\text {mdeb }}$ sont respectivement l'azote minéral du profil en fin et en début de culture sur parcelle nue (moyenne des 2 prélèvements rapprochés), $N_{\text {mmaïs }}$ l'azote minéral du profil sous maïs à la récolte et $N_{\text {exp }}$ l'azote exporté par les parties aériennes à la récolte $\left(\mathrm{kg} \cdot h \mathrm{a}^{-1}\right)$. $R$ est le rapport entre l'azote organisé dans le sol sous maïs et les exportations par les parties aériennes avec l'hypothèse que ce rapport est le même pour les différentes situations.

\section{RÉSULTATS}

Les résultats présentés dans la figure 1 font apparaître une liaison étroite entre la minéralisation nette de l'azote en incubation à $20^{\circ} \mathrm{C}$ et à l'extérieur ( $r=0,974 \mathrm{ddl})$. Le fait que seules les températures et la durée d'incubation diffèrent dans les 2 expériences explique cette bonne corrélation. On peut donc prendre indifféremment l'un ou l'autre test comme référence aux potentialités des sols à minéraliser.

L'azote minéral présent dans le profil sur la période de culture du maïs figure dans le tableau II. On peut noter l'importance des reliquats fin avril-début mai $\left(70-135 \mathrm{~kg}^{\circ} \mathrm{ha}^{-1}\right)$ attribuables à la minéralisation des résidus du précédent, à l'azote minéral non utilisé par la culture qui précède et à un début de minéralisation endogène au printemps.

En parcelle nue, l'hétérogénéité de répartition de l'azote minéral dans le sol (tableau II) est plus faible que celle observée par Lindemann (1986); ceci tient au fait que chaque mesure porte sur un échantillon moyen de 4 prélèvements par horizon. Pour les parcelles 2 , 4, et 6 une partie importante de l'azote minéral en

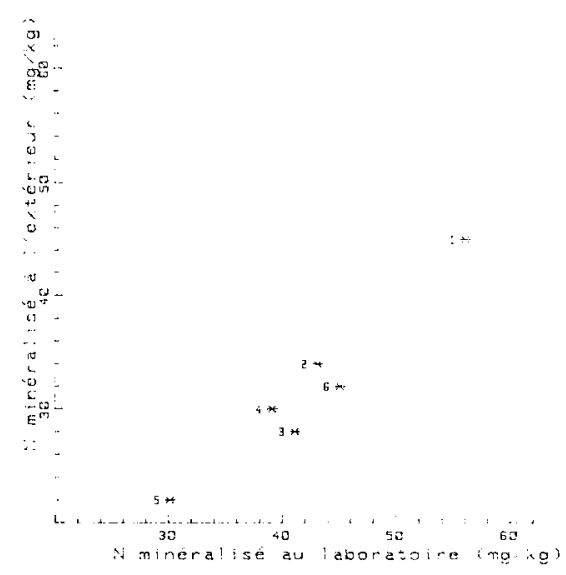

Fig 1. Relation entre la minéralisation nette de l'azote des 6 sols après incubation au laboratoire à $20^{\circ} \mathrm{C}(25-300 \mathrm{j})$ et à l'extérieur (de mai à décembre 1989). 
Tableau II. Azote minéral dans le profil et exportation par le maïs ON au stade ensilage ( $\mathrm{kg} / \mathrm{ha}$ ).

\begin{tabular}{|c|c|c|c|c|c|c|c|}
\hline \multirow[b]{3}{*}{ Date de prél } & \multicolumn{5}{|c|}{$N$ minéral du profil parcelles nues oN } & \multicolumn{2}{|c|}{ Parcelles maïs $\mathrm{ON}$} \\
\hline & & & & & & $N_{\exp }^{*}$ & $N_{\text {mmais }}{ }^{* *}$ \\
\hline & 27 avril & $12 \mathrm{mai}$ & 28 juil & 6 sept & 15 sept & 8 sept & 15 sept \\
\hline Site $n^{\circ} 1$ & $109(15)$ & $99(11)$ & $188 \quad(7)$ & $246(20)$ & $229(12)$ & $139(25)$ & $23(3)$ \\
\hline Site $n^{\circ} 2$ & $137(28)$ & $121(48)$ & $123(27)$ & $148(26)$ & $127(26)$ & $99(10)$ & $32(4)$ \\
\hline Site $n^{\circ} 3$ & $73(5)$ & $70(6)$ & $140 \quad(2)$ & $153(16)$ & $145(16)$ & 101 & $24(2)$ \\
\hline Site $n^{\circ} 4$ & $114(6)$ & 104 & $130(6)$ & $148(20)$ & 159 & $94(12)$ & $26(3)$ \\
\hline Site $n^{\circ} 5$ & 71 (2) & $72(7)$ & $68 \quad$ (3) & $82(10)$ & $73(5)$ & $87(11)$ & $15(1)$ \\
\hline Site $n^{\circ} 6$ & $105(10)$ & 108 & $98 \quad(5)$ & $112(4)$ & $102 \quad(4)$ & $70(6)$ & $4(1)$ \\
\hline
\end{tabular}

* $N$ parties aériennes (feuille, tige et grain); ${ }^{\star \star} \mathrm{N}$ minéral du profil à la récolte; ( ) écart type.

début de culture est localisée en profondeur (fig 2), avec par conséquent des risques de lixiviation des nitrates, surtout en sol nu. La variabilité à court terme (2 premières et 2 dernières mesures) est de l'ordre de grandeur de la variabilité au champ. On n'observe pas, dans nos conditions expérimentales, les variations importantes d'azote minéral du sol dans le temps attribuées à des cycles rapides de minéralisationréorganisation (Hébert, 1965).

À plus long terme, l'évolution de l'azote minéral (tableau II) se manifeste par une augmentation dans le temps pour les parcelles 1,3 et 4 alors que pour les parcelles 2,5 et 6 , on n'observe pas d'augmentation apparente sur tout le profil. Sur la parcelle 2, l'augmentation dans les horizons de surface est compensée par une baisse en profondeur (fig 2).

La matière sèche produite respectivement pour les parcelles $1-6$ est la suivante $\left(\mathrm{t}(\mathrm{MS}) \cdot \mathrm{ha}^{-1}\right)$ : $15,0-11,0-12,4-12,9-10,1-9,8$. Le reliquat d'azote après maïs (tableau II) varie de 15 à 30 $\mathrm{kg}^{\circ} \mathrm{ha}^{-1}$, valeurs considérées comme non extractibles par la culture (Rémy et Hébert, 1977). Le faible reliquat sur le site 6 est dû au fait que la parcelle maïs a une épaisseur de sol plus faible $(50-60 \mathrm{~cm})$ que celle maintenue nue $(80 \mathrm{~cm})$.

\section{DISCUSSION}

À condition que la culture ne modifie pas la minéralisation endogène, on peut considérer que l'azote minéral présent dans le profil sous sol nu en automne $\left(N_{\text {mfin }}\right)$ et les prélèvements par les parties aériennes $\left(N_{\text {exp }}\right)$ rendent compte respec- tivement des disponibilités et de l'utilisation de ces disponibilités. Le rapport $\left(\mathrm{N}_{\text {mfin }}-\mathrm{N}_{\text {mmais }}\right)$ / $\left(N_{\text {exp }}\right)$ varie de 1,2 à 1,5 pour les parcelles 1, 3, 4 et 6 . Ces valeurs peuvent être considérées conformes à celles $(1,2-1,25)$ obtenues en conditions contrôlées (Hétier et al, 1986) si on tient compte des imprécisions statistiques et de la variabilité vraisemblable de ce rapport selon les conditions de milieu et le niveau de fertilisation de la culture. On peut admettre que, pour ces parcelles, il n'y a pas de distorsion entre les 2 méthodes au champ. Pour les parcelles 2 et 5 , en revanche, les valeurs faibles de ce rapport (respectivement 1,1 et 0,7 ) résulteraient d'une sous-estimation de la fourniture d'azote par le sol en parcelle nue.

L'azote minéral de la parcelle nue en automne et celui exporté par le maïs à la récolte proviennent à la fois du reliquat d'azote au semis et de la minéralisation sur la durée de la culture. La production d'azote minéral des essais d'incubation rend surtout compte, en revanche, de la minéralisation du sol. Pour pouvoir comparer ces 2 types de résultats, on est amené :

- à prendre en compte le bilan d'azote minéral au champ entre le début et la fin de la culture;

- à admettre une même proportionalité pour les différents sols entre la fourniture d'azote par la couche arable (supposée de même épaisseur) et le sous-sol;

- à supposer que la proportion d'azote prélevé par le maïs et mobilisé dans les racines et la biomasse microbienne associée est identique pour les différentes parcelles.

Les erreurs inhérentes au calcul de bilans et le caractère plus ou moins fondé des hypothèses 

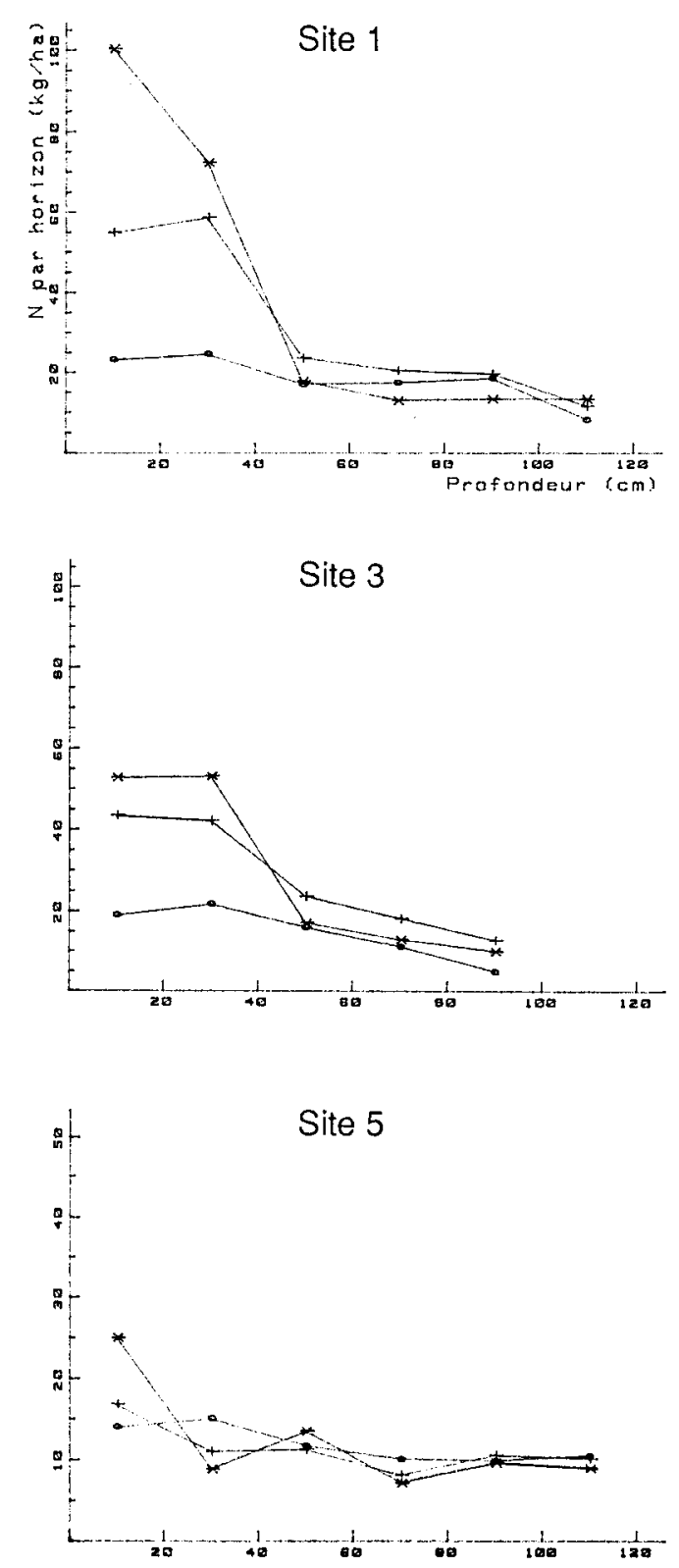
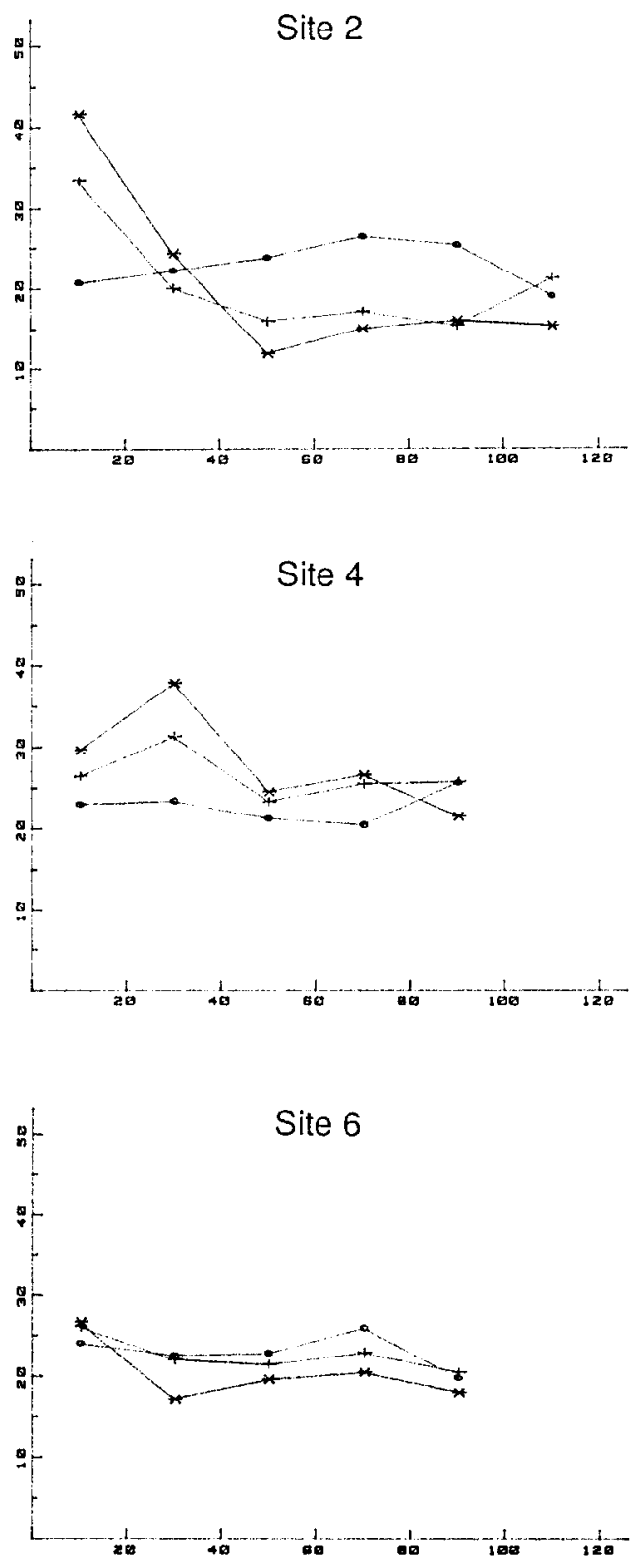

Fig 2. Évolution des profils d'azote minéral sous sol nu dans les 6 sites. $0: 27$ avril $1989,+: 25$ juillet $1989,{ }^{\star}: 15$ septembre 1989.

avancées limitent la précision de la comparaison des 2 méthodes d'estimation de la minéralisation endogène.

Ces restrictions faites, on peut noter que (fig $3)$, indépendamment des écarts déjà signalés entre les résultats obtenus en sol nu et sur maïs oN, pour les parcelles 1,3 et 5 , il y a cohérence entre les déterminations en conditions contrôlées et au champ, alors que pour les parcelles 2,4 et 6 , le potentiel apparaît nettement supérieur à son expression sur le terrain. Un certain nombre d'hypothèses peuvent être avancées pour expliquer ces différences :
- une moindre participation du sous-sol en raison de teneurs en matières organiques plus basses ou d'une profondeur plus faible;

- une réduction de l'activité microbienne consécutive à des humidités faibles ou à des échanges gazeux restreints en rapport avec la porosité des sols;

- des pertes par lixiviation envisageables principalement sur les parcelles nues irriguées;

- des pertes par dénitrification, surtout en sol nu, en raison de la fréquence d'humidités élevées et éventuellement sous maiis. 


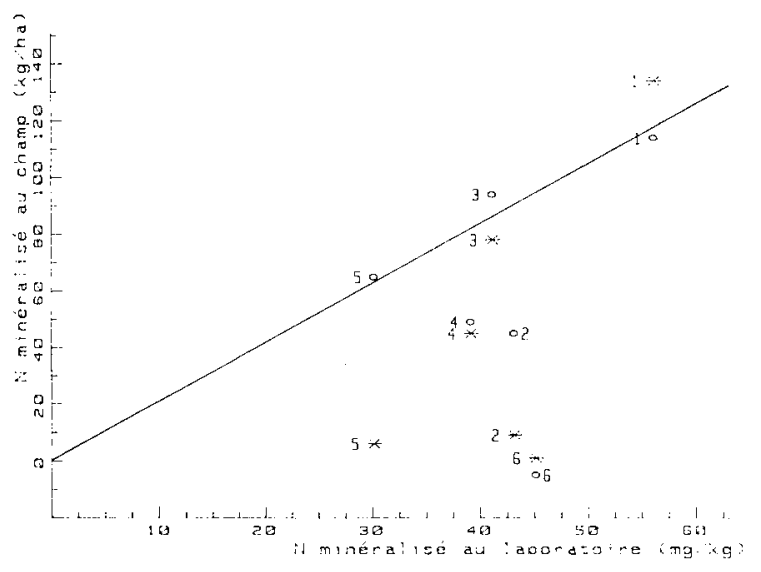

Fig 3. Relation entre le pouvoir minéralisateur (25-300 j) à $20^{\circ} \mathrm{C}$ et la minéralisation au champ. * : parcelle nue : minéralisation estimée par l'équation $1 ; 0$ : parcelle maïs : minéralisation estimée par l'équation $2, R=0,4$.

La part du sous-sol dans la fourniture d'azote minéral par le profil est souvent faible, $20 \%$ selon Rémy et Hébert (1977); nous observons nous-mêmes, sur des tests d'incubation au laboratoire, que l'azote produit par le sous-sol de la parcelle 1 en conditions d'aération non limitante représente $17 \%$ de celui de la couche arable. C'est surtout pour la parcelle 6 (nu et maïs), moins profonde, qu'un effet sous-sol peut être envisagé. Les effets de l'humidité sur l'activité microbienne ont été surtout étudiés en laboratoire. Dans la gamme des teneurs en eau observées sous culture, la réduction d'activité n'excéderait pas 20-30\% (Stanford et Epstein, 1974) et ne peut être envisagée que pour les parcelles sous maïs non irriguée (1 et 2). II est vraisemblable que, compte tenu de l'importance des écarts observés, la porosité des sols soit également impliquée. L'examen des profils nitriques des différents prélèvements fait apparaître des teneurs plus élevées en profondeur pour les parcelles 2, 4 et 6 que pour les autres (fig 2); dans ces situations, les risques de percolation des nitrates en profondeur pourraient être plus élevés sur parcelles nues irriguées et plus limités sous maïs selon l'importance de l'irrigation et l'épaisseur du sol. Les pertes par dénitrification sous cultures, lorsqu'elles sont déterminées directement, sont en général faibles (Germon et Couton, 1989); sous maïs non fertilisé, les pertes sont négligeables (Duxbury et Mc Connaughey, 1986). En sol nu, les données disponibles sont rares, des mesures de dénitrification par blocage à l'acéthylène faites sur une parcelle voisine du sol 1 donnent des pertes en été de $6 \mathrm{~kg}(\mathrm{~N}) \cdot \mathrm{ha}^{-1}$ (Lehn-Reiser et al, 1989). On peut envisager, néanmoins, qu'en parcelle nue, dans le cas de sols moins filtrants, le phénomène puisse être plus important (sites 2 et 5 ).

D'un point de vue méthodologique, l'estimation de la minéralisation endogène nette au champ est difficile; elle repose sur des bilans d'azote portant sur des valeurs souvent hétérogènes. L'augmentation du nombre d'échantillons sur le terrain permet de remédier à cette situation, mais les moyens à mettre en œuvre deviennent rapidement excessifs. Parmi les méthodes d'estimation de la fourniture d'azote par le sol au champ (Taureau, 1987), le suivi d'azote minéral sur parcelle nue non fertilisée intervient dans des conditions hydriques parfois éloignées de celles qui prévalent sous culture et qui peuvent favoriser les pertes d'azote (lixiviation et éventuellement dénitrification) ou ralentir l'activité minéralisatrice. Si la culture sans fertilisation azotée offre des conditions de sol proches - hormis les disponibilités en azote - de celles au champ et peut être prise de ce fait comme référence, la part de l'azote allouée aux racines demeure une source d'imprécisions. Le recours au $15 \mathrm{~N}$ peut apporter une amélioration à condition de mesurer le ${ }^{15} \mathrm{~N}$ minéral et organique restant dans le sol après récolte, ce qui rend la méthode coûteuse.

Une étude antérieure (Delphin, 1986) a montré qu'on pouvait estimer avec une précision satisfaisante le pouvoir minéralisateur des sols à partir de caractéristiques physico-chimiques courantes. Dans la relation (fig 3) entre l'azote produit au laboratoire sur la période 25-300 j (potentialités) et l'estimation de la minéralisation au champ par les 2 méthodes (expression des potentialités), l'équation de l'enveloppe externe du nuage de points passant par l'origine $(Y=2,1 X)$ permet, en première approximation, d'établir la correspondance entre les potentialités et leur expression au champ, pour la culture du maïs, pour les conditions climatiques de l'année 1989 et sans intervention de facteurs limitants importants. Cependant, pour être utilisé dans la pratique, ce type de résultats devrait être complété par la caractérisation des processus et des conditions de milieux impliqués dans la réduction de ces potentialités.

\section{CONCLUSION}

La comparaison des mesures de minéralisation au laboratoire et au champ, compte tenu des biais inhérents aux méthodes de terrain, montre que, dans certaines situations, la fourniture d'azote par le sol est nettement inférieure aux 
potentialités. Le transfert de nitrates en profondeur peut expliquer en partie ces résultats; il est également probable que les conditions d'humidité et de porosité à l'air soient à l'origine d'une réduction de l'activité microbienne et éventuellement induisent une dénitrification. Des mesures directes au champ devraient permettre de vérifier ces hypothèses. Pour pouvoir estimer l'azote fourni par minéralisation et disponible pour une culture à partir du pouvoir minéralisateur des sols apprécié en laboratoire, il est nécessaire de connaître la nature et l'importance des facteurs de milieu susceptibles d'entraver l'expression au champ de ces potentialités. L'élaboration d'un conseil de fumure pertinent visant à limiter les excès d'azote en agriculture doit donc s'appuyer sur une caractérisation fiable et précise du milieu dans lequel les systèmes de culture sont mis en œuvre.

\section{REMERCIEMENTS}

Cette étude a été en partie financée par l'Association générale des producteurs de maïs (AGPM).

\section{RÉFÉRENCES}

Chaussod R, Nicolardot B, Catroux G, Chrétien J (1986) Relations entre les caractéristiques physicochimiques et microbiennes de quelques sols cultivés. Sci Sol 2, 213-226

Delphin JE (1986) Évaluation du pouvoir minéralisateur de sols agricoles en fonction de leurs caractéristiques physico-chimiques. Agronomie 6, 453-458

Duxbury JM, Mc Connaughey PK (1986) Effect of fertilizer source on denitrification and nitrous oxide emission in a maize-field. Soil Sci Soc Am J 50, 644-648

Germon JC, Couton $Y$ (1989) Nitrogen balance and gazeous nitrogen losses in soils. In: Management systems to reduce impact of nitrates. (JC Germon, ed) Elsevier Appl Sci
Hébert J (1965) Observation de la minéralisation de l'azote en sol nu. CR Séances Acad Agric Fr 51, 850-858

Hétier JM, Andreux F, Schouller E, Marol C (1986) Organic matter inputs to soil after growth of Carbon 14 - Nitrogen 15 labeled maize. Soil Sci Soc Am J $50,76-80$

Jenkinson DS, Rayner JH (1977) The turnover of soil organic matter in some of the Rothamsted classical experiment. Soil Sci 123, 298-305

Lehn-Reiser M, Munch JC, Chapot JY, Ottow JCG (1990) Field measured denitrification losses from a calcerous inceptisol after green manuring. Mitt $D$ Bodenk Gesellschaft 60, 233-238

Lindemann $Y$ (1986) Contribution à l'étude statistique des répartitions et à la modélisation de la dynamique de l'azote nitrique dans le sol. Thèse Univ Paris-Sud, $310 \mathrm{p}$

Mary B (1988) Rôle de la biomasse microbienne du sol dans la disponibilité en azote minéral en conditions de plein champ. CR SRETIE, Ministère de l'Environnement, $82 \mathrm{p}$

Mary B, Rémy JC (1979) Essai d'appréciation de la capacité de minéralisation de l'azote des sols de grande culture. I. Signification des cinétiques de minéralisation de la matière organique humifiée. Ann Agron 30, 513-527

Meisinger JJ (1984) Evaluating plant-available nitrogen in soil-crop systems. In: Nitrogen in crop production. (RD Hauck et al, eds) Am Soc Agron Madison, WI, 391-416

Rémy JC, Hébert J (1977) Le devenir des engrais azotés dans le sol. CR Séances Acad Agric Fr 163, 700-714

Stanford G, Epstein E (1974) Nitrogen mineralization - water relations in soil. Soil Sci Soc Am Proc 38, 103-106

Taureau JC (1987) Approche de l'offre en azote du milieu par les expérimentations au champ. $C R$ Séances Acad Agric Fr 73, 81-90

Taureau JC, Desvignes $P$ (1990) Fourniture du sol et coefficient d'efficacité : deux points clés de la méthode du bilan. Application au blé et au maïs. In: Nitrates, agriculture, eau. ( $R$ Calvet, ed) Paris, 7-8 novembre $1990,467-474$ 